\author{
Military Technical College \\ Kobry El-Kobbah, \\ Cairo, Egypt.
}

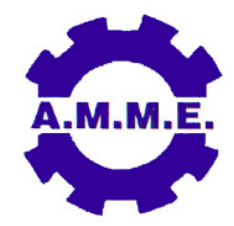

\title{
BUOYANCY DRIVEN FLOW WITHIN UNIFORMLY HEATED ECCENTRIC ANNULUS
}

\author{
F. M. Mahfouz ${ }^{*}$ and R. M. Rizk-Allah**
}

\begin{abstract}
The buoyancy driven flow within uniformly heated eccentric annulus has been numerically predicted. The annulus that formed between two eccentric circular cylinders is placed horizontally and its inner wall is heated at uniform heat flux while its outer wall is isothermally cooled. The results for induced laminar natural convection flow were carried out at modified Rayleigh number of $10^{4}$ and at different values of outer to inner cylinder radius ratio, eccentricity and angle of inclination of annulus symmetry line (ASL) to the horizontal. The results for local and average temperature of the inner wall are obtained and discussed together with the details of both flow and thermal fields. The study has predicted the global flow circulation around the inner cylinder. The study has shown that the steady average temperature of inner wall decreases for negative eccentricities while it increases for same but positive eccentricities. The study has also shown that as the angle of inclination of ASL increases from 0 to 90 the steady average temperature of the inner wall decreases for negative eccentricities while it increases for same but positive eccentricities.
\end{abstract}

\section{KEY WORDS}

Natural Convection, Constant Heat Flux, Fourier Spectral Method, Analytical solution

\footnotetext{
* Professor, Dept. of Mech. Power, Faculty of Engineering, Menoufia Univ., Egypt.
}

** Lecturer, Dept. of Basic sciences, Faculty of Engineering, Menoufia Univ., Egypt. 


\section{NOMENCLATURE}

ASL Annulus symmetry line

$B_{0}, B_{n} \quad$ coefficients that result from the expansion of $-J^{1 / 2}$

$\mathrm{C}$

d $\quad \mathrm{c} / \mathrm{Ri}_{\mathrm{i}}$

E eccentricity

e dimensionless eccentricity $=\mathrm{E} /\left(R_{o}-R_{i}\right)$

$f_{n}, F_{n} \quad$ Fourier coefficients

$\mathrm{Fb} \quad$ buoyancy force

g gravitational acceleration

$g_{n}, G_{n} \quad$ Fourier coefficients

$\mathrm{h}_{\mathrm{n}}, \mathrm{H}_{\mathrm{n}} \quad$ Fourier coefficients

$J$

k Jacobian of coordinate transformation

thermal conductivity

$\mathrm{L} \quad$ radius difference $=\left(R_{o}-R_{i}\right)$

$\operatorname{Pr} \quad$ Prandtl number $(=\nu / \alpha)$

$\dot{Q} \quad$ heat transfer rate

q heat flux

$\mathrm{q}_{\mathrm{r}} \quad$ heat flux ratio $\left(\mathrm{q}_{\mathrm{o}} / \mathrm{q}_{\mathrm{i}}\right)$

$R_{0}, R_{i} \quad$ outer and inner radius of the annulus

$\mathrm{Rr} \quad$ radius ratio $=\left(R_{o} / R_{i}\right)$

RaL Rayleigh number based on $\mathrm{L}\left(=\mathrm{g} \beta(L)^{4} q / k \alpha v\right)$

$\mathrm{t} \quad$ dimensionless time

$T \quad$ temperature

THR Total heat transfer ratio

$\mathrm{x}^{\prime}, \mathrm{y}^{\prime} \quad$ Cartesian coordinates

$\mathrm{x}, \mathrm{y}$ dimensionless Cartesian coordinates

\section{Greek symbols}

$\begin{array}{ll}\alpha & \text { thermal diffusivity } \\ \beta & \text { coefficient of thermal expansion } \\ \phi & \text { dimensionless temperature }=k\left(T-T_{o}\right) / R_{i} q_{i} \\ \eta, \xi & \text { Bi-polar coordinates } \\ \lambda & \text { angle of inclination of ASL measured from horizontal } \\ \nu & \text { kinematics viscosity } \\ \rho & \text { density } \\ \theta & \text { Polar angle measured from ASL. } \\ \tau & \text { time }\end{array}$


$\psi^{\prime}, \psi \quad$ dimensional and dimensionless stream functions

$\zeta^{\prime}, \zeta \quad$ dimensional and dimensionless vorticity

\section{Subscripts}

$\begin{array}{ll}i & \text { at the inner wall } \\ 0 & \text { at the outer wall }\end{array}$

\section{INTRODUCTION}

The problem of natural convection within an annular space between two concentric/eccentric cylinders has long been of both academic and practical interest because of its numerous engineering applications. These applications include solar collector-receivers, high-voltage electric transmission cables, cooling systems in nuclear reactors, and many others. Many experimental and numerical studies in case of natural convection between two concentric cylinders has been conducted. A good review of the relevant literatures has been given by Kuehn and Goldstein [1].

In comparison with the concentric case, the volume of studies on natural convection between two eccentric cylinders is relatively small. Most of these studies have mainly focused on the annulus between two vertically eccentric or horizontally eccentric cylinders. Kuehn and Goldstein [2], Chakrabarti et al. [3], Prusa and Yao [4], Habibi and Pop [5], to mention a few, studied the natural convection in case of vertically eccentric annulus while Guj and Stella [6] and Guj et al. [7] studied the thermal field in horizontally eccentric annulus. In case of vertical eccentricity the annulus symmetry line, ASL (the line that connects the center of inner cylinder to the center of outer cylinder) is parallel to the gravity vector and hence the resulting flow field is symmetric with respect to the ASL and only half of the annulus can be considered as the computational domain. As a consequence, the authors used to set the boundary conditions for stream function to zero on both the inner wall and outer wall. Such a treatment can not be used in case of ASL being inclined due to the possibility of having global flow circulation around the inner wall of the annulus.

The possibility of having natural global flow circulation within annular enclosures increases in the enclosures which are not symmetric with respect to gravity vector. Previously, Mahfouz [8] has been predicted numerically such a global flow in case of inclined elliptic enclosure. Some other authors have also investigated the possibility of having the global flow in asymmetric annuli. Kuehn and Goldstein [2] experimentally predicted the global flow using the interferograms which were not clear enough to confirm whether the global circulation exists or not. Wang and Bau [9] also studied the flow field using a perturbation expansion. On the other side, Cho et al. [10] and Projahn et al. [11] investigated numerically the problem using vorticitystream function formulation, but they assigned zero values to the stream function on the two walls of the annulus which prevents the numerical prediction of the global flow in the annulus. This treatment is a good approximation only in the case of small eccentricity but can not be used for large eccentricities where the stream function 
values on the two walls are quite different because of the global flow circulation [12, 13].

Natural convection in case of asymmetric annuli has been further considered by a number of authors who applied different numerical techniques to investigate the validity of such techniques to solve the problem. Shu et al. [14] used polynomialbased differential quadrature (PDQ) and Fourier expansion-based differential quadrature (FDQ) while Shu and Wu [15] used the domain-free discretization (DFD) method. Lee et. al. [16] used a generalized differential-integral quadrature (GDQ) technique to discretize the natural convection governing equations using a body-fitted coordinate system. Recently, Mahfouz [17] used Fourier Spectral Method (FSM) to solve the same problem.

In case of heating the annulus at constant heat flux, only few studies were reported on buoyancy driven fluid flow in annular enclosures. These studies considered the cases of symmetrical configuration in which no global flow circulation is reported. Castrejon and Spalding [18], Yoo [19, 20] have considered the case of annulus between two concentric tubes while Glakpe et al. [21], and Ho et al. [22] considered the case of eccentric annulus.

It can be observed that most of the previous studies focused on the steady natural convection within vertically symmetric eccentric annuli. In case of natural convection within asymmetric annuli most of the studies have focused on isothermally heated annuli through the inner wall. Moreover, such studies give more care to plotting the flow and thermal fields without paying enough attention to the effect of inclination of ASL on thermal field at different eccentricities. Therefore, the objective of this study is to develop an accurate numerical technique, using bi-polar orthogonal coordinate transformation together with the Fourier Spectral Method (FSM), to further extend the existing knowledge on natural convection heat transfer in arbitrarily oriented eccentric annuli and heated at constant heat flux.

\section{PROBLEM FORMULATION}

The physical domain and coordinate systems are shown Fig.1. The annulus under consideration, as shown in the figure, is the 2-D space formed between two long horizontal eccentric cylinders. The outer cylinder has radius $R_{o}$, while the inner cylinder has radius $R_{i}$, and is placed in such away the plane passing through its center and through the center of outer cylinder inclines with angle $\lambda$ to the horizontal. The eccentricity, $\mathrm{E}$ between the two cylinders is taken positive if the center of the inner cylinder is above that of the outer cylinder ( or to the right of it ) and vice versa. The annulus contains incompressible, Newtonian fluid and is heated through its inner wall at constant heat flux, $q_{i}$. The annulus outer wall is cooled and kept at constant temperature $T_{o}$. The resulting buoyancy driven flow in the annulus is assumed to be laminar and two dimensional. The density of the fluid is considered constant except in the buoyancy term where it varies according to Boussinesq approximation. Moreover, viscous dissipation, internal heat generation and radiation heat transfer are either absent or neglected. 
Immediately, after heating the annulus through its inner wall a buoyancy driven flow is initiated. The induced flow has been initially time dependent and the steady state condition is reached after a period of time. The time dependent conservation equations of mass, momentum and energy can be written in Cartesian coordinates in terms of stream function, vorticity and temperature as:

$$
\begin{gathered}
\frac{\partial \zeta^{\prime}}{\partial \tau}=v\left(\frac{\partial^{2} \zeta^{\prime}}{\partial x^{\prime 2}}+\frac{\partial^{2} \zeta^{\prime}}{\partial y^{\prime 2}}\right)-\frac{\partial \psi^{\prime}}{\partial y^{\prime}} \frac{\partial \zeta^{\prime}}{\partial x^{\prime}}+\frac{\partial \psi^{\prime}}{\partial x^{\prime}} \frac{\partial \zeta^{\prime}}{\partial y^{\prime}}-\frac{1}{\rho}\left(\frac{\partial F_{x^{\prime}}}{\partial y^{\prime}}-\frac{\partial F_{y^{\prime}}}{\partial x^{\prime}}\right) \\
\zeta^{\prime}+\left(\frac{\partial^{2} \psi^{\prime}}{\partial x^{\prime 2}}+\frac{\partial^{2} \psi^{\prime}}{\partial y^{\prime 2}}\right)=0 \\
\frac{\partial T}{\partial \tau}=\alpha\left(\frac{\partial^{2} T}{\partial x^{\prime 2}}+\frac{\partial^{2} T}{\partial y^{\prime 2}}\right)-\frac{\partial \psi^{\prime}}{\partial y^{\prime}} \frac{\partial T}{\partial x^{\prime}}+\frac{\partial \psi^{\prime}}{\partial x^{\prime}} \frac{\partial T}{\partial y^{\prime}}
\end{gathered}
$$

where $\tau$ is the time, $\psi^{\prime}$ is the stream function, $\zeta^{\prime}$ is the vorticity, $T$ is the temperature, $\rho$ is the density, $v$ and $\alpha$ are the momentum and thermal diffusivities respectively. $F_{x^{\prime}}=\rho g \beta\left(T-T_{o}\right) \sin (\lambda), F_{y^{\prime}}=\rho g \beta\left(T-T_{o}\right) \cos (\lambda)$ are the components of the net upward driving force, $F_{b}$.

The fluid inside the annulus is assumed initially stagnant and at a temperature equal to that of outer wall. While the inner wall is assumed to be impulsively heated and maintained at uniform heat flux. The hydrodynamics boundary conditions at the inner and outer walls are the no-slip condition and impermeability. The thermal boundary conditions at the inner and outer walls are the constant heat flux and constant wall temperature, $q_{i}$, and $T_{o}$, respectively. The steady state boundary conditions can be expressed mathematically as follows:

- On the inner wall

$$
\frac{\partial \psi^{\prime}}{\partial x^{\prime}}=0, \frac{\partial \psi^{\prime}}{\partial y^{\prime}}=0, \text { and } \quad q_{i}=-k \frac{\partial T}{\partial S_{n}}=\text { const } .
$$

-On the outer wall

$$
\psi^{\prime}=\frac{\partial \psi^{\prime}}{\partial x^{\prime}}=0, \quad \frac{\partial \psi^{\prime}}{\partial y^{\prime}}=0 \quad \text { and } T=T_{o}
$$

The stream function, as indicated above, is set to zero on the outer wall while its value on the inner wall will be calculated. The calculated value of the stream function at the inner wall will determine both intensity and direction of the global flow circulation around the inner wall, if exists. 


\section{Coordinate Transformation}

In order to set properly the inner and outer walls boundary conditions without interpolation, a coordinate system that makes the boundary lines coincide with coordinates lines should be used. In this problem the bipolar coordinates $\xi, \eta$ defined as $x=-d \sinh \xi /(\cosh \xi-\cos \eta), \quad y=d \sin \eta /(\cosh \xi-\cos \eta)$ are adopted. In bipolar coordinates the governing equations (1-3) read:

$$
\begin{gathered}
J \frac{\partial \zeta}{\partial t}=\operatorname{Pr}\left(\frac{\partial^{2} \zeta}{\partial \xi^{2}}+\frac{\partial^{2} \zeta}{\partial \eta^{2}}\right)+\left(\frac{\partial \psi}{\partial \xi} \frac{\partial \zeta}{\partial \eta}-\frac{\partial \psi}{\partial \eta} \frac{\partial \zeta}{\partial \xi}\right) \\
+\frac{\operatorname{Pr} R a L}{d^{*}(R r-1)^{4}} \sin (\lambda)\left[J(\cosh \xi \cos \eta-1) \frac{\partial \phi}{\partial \eta}-J\left(\sinh \xi \sin \eta \frac{\partial \phi}{\partial \xi}\right]\right. \\
-\frac{\operatorname{Pr} R a L}{d^{*}(R r-1)^{4}} \cos (\lambda)\left[J(\cosh \xi \cos \eta-1) \frac{\partial \phi}{\partial \xi}+J\left(\sinh \xi \sin \eta \frac{\partial \phi}{\partial \eta}\right]\right. \\
J \zeta=\frac{\partial^{2} \psi}{\partial \xi^{2}}+\frac{\partial^{2} \psi}{\partial \eta^{2}} \\
J \frac{\partial \phi}{\partial t}=\left(\frac{\partial^{2} \phi}{\partial \xi^{2}}+\frac{\partial^{2} \phi}{\partial^{2} \eta}\right)+\frac{\partial \psi}{\partial \xi} \frac{\partial \phi}{\partial \eta}-\frac{\partial \psi}{\partial \eta} \frac{\partial \phi}{\partial \xi}
\end{gathered}
$$

where

$x=x^{\prime} / R_{i}, y=y^{\prime} / R_{i}, \quad d=c / R_{i}, \quad t=\tau \alpha / R_{i}^{2}, \quad \psi=\psi^{\prime} / \alpha, \quad \zeta=-\zeta^{\prime} R_{i}^{2} / \alpha \quad$ and $\phi=k\left(T-T_{o}\right) / R_{i} q_{i} . J=x_{\xi} y_{\eta}-x_{\eta} y_{\xi}$ is the determinant of the Jacobian of coordinate transformation matrix, $\operatorname{Pr}=v / \alpha$ is the Prandtl number, $R a L=g \beta R_{i}^{4}(R r-1)^{4} q_{i} / k v \alpha$ is the Rayleigh number.

In the new coordinates, the steady boundary conditions (4) can now be expressed as:

- On the inner cylinder surface, $\xi=\xi_{i}$

$$
\frac{\partial \psi}{\partial \xi}=0, \frac{\partial \psi}{\partial \eta}=0 \quad \text { and } \quad \frac{\partial \phi}{\partial \xi}=-J^{1 / 2}
$$

- On the outer cylinder surface, $\xi=\xi_{\text {o }}$

$$
\psi=\frac{\partial \psi}{\partial \xi}=0, \frac{\partial \psi}{\partial \eta}=0 \quad \text { and } \quad \phi=0
$$

where $\xi_{i}$ and $\xi_{o}$ are negative constants that define the inner and outer walls surfaces $\left(\xi_{i}=\sinh ^{-1}(-d), \xi_{o}=\sinh ^{-1}(-d / R r)\right)$ where $R r=R_{o} / R_{i}$ is the radius ratio. 


\section{METHOD OF SOLUTION}

The method of solution is based on Fourier spectral method in which the dependent variables stream function $\psi$, vorticity $\zeta$ and temperature $\phi$ are approximated using Fourier series expansion. The approach is similar to that used by Badr and Dennis [23] and Mahfouz and Badr [24]. The dimensionless stream function, vorticity and temperature are approximated as:

$$
\begin{gathered}
\psi(\xi, \eta, t)=\frac{1}{2} \mathrm{~F}_{\mathrm{o}}(\xi, t)+\sum_{n=1}^{N}\left[f_{n}(\xi, t) \sin (n \eta)+F_{n}(\xi, t) \cos (n \eta)\right] \\
\zeta(\xi, \eta, t)=\frac{1}{2} \mathrm{G}_{\mathrm{o}}(\xi, t)+\sum_{n=1}^{N}\left[g_{n}(\xi, t) \sin (n \eta)+G_{n}(\xi, t) \cos (n \eta)\right] \\
\phi(\xi, \eta, t)=\frac{1}{2} \mathrm{H}_{\mathrm{o}}(\xi, t)+\sum_{n=1}^{N}\left[h_{n}(\xi, t) \sin (n \eta)+H_{n}(\xi, t) \cos (n \eta)\right]
\end{gathered}
$$

where $F_{o}, f_{n}, F_{n}, G_{o}, g_{n}, G_{n}, H_{o}, h_{n}$, and $H_{n}$ are the Fourier coefficients and $N$ represents the number of terms considered in the Fourier series. Substitution of $\psi$, $\zeta$ and $\phi$ defined in (9) in equations (5-7) and integrating these equations (after multiplying each by $1, \sin (n \eta)$ and $\cos (n \eta)$ respectively) from 0 to $2 \pi$ results in the following set of differential equations:

$$
\begin{gathered}
\frac{\partial^{2} F_{o}}{\partial \xi^{2}}=a_{o} G_{o}+\sum_{1}^{N} a_{m} G_{m} \\
\frac{\partial^{2} F_{n}}{\partial \xi^{2}}-n^{2} F_{n}=\frac{1}{2} a_{n} G_{o}+a_{o} G_{n}+\frac{1}{2} \sum_{1}^{N} G_{m}\left(a_{n+m}+a_{|m-n|}\right) \\
\frac{\partial^{2} f_{n}}{\partial \xi^{2}}-n^{2} f_{n}=a_{o} g_{n}+\frac{1}{2} \sum_{1}^{N} g_{m}\left(a_{|m-n|}-a_{n+m}\right) \\
a_{o} \frac{\partial G_{o}}{\partial t}+\sum_{1}^{N} a_{m} \frac{\partial G_{m}}{\partial t}=\operatorname{Pr} \frac{\partial^{2} G_{o}}{\partial \xi^{2}}+S G_{o}(\xi, t) \\
a_{o} \frac{\partial G_{n}}{\partial t}+\frac{1}{2} \frac{\partial G_{o}}{\partial t} a_{n}+\frac{1}{2} \sum_{1}^{N} \frac{\partial G_{m}}{\partial t}\left(a_{n+m}+a_{|n-m|}\right)=\operatorname{Pr}\left(\frac{\partial^{2} G_{n}}{\partial \xi^{2}}-n^{2} G_{n}\right)+S G_{n}(\xi, t) \\
a_{o} \frac{\partial g_{n}}{\partial t}+\frac{1}{2} \sum_{1}^{N} \frac{\partial g_{m}}{\partial t}\left(a_{|m-n|}-a_{n+m}\right)=\operatorname{Pr}\left(\frac{\partial^{2} g_{n}}{\partial \xi^{2}}-n^{2} g_{n}\right)+S g_{n}(\xi, t) \\
a_{o} \frac{\partial H_{o}}{\partial t}+\sum_{1}^{N} a_{m} \frac{\partial H_{m}}{\partial t}=\frac{\partial^{2} H_{o}}{\partial \xi^{2}}+Z H_{o}(\xi, t)
\end{gathered}
$$




$$
\begin{gathered}
a_{o} \frac{\partial H_{n}}{\partial t}+\frac{1}{2} \frac{\partial H_{o}}{\partial t} a_{n}+\frac{1}{2} \sum_{1}^{N} \frac{\partial H_{m}}{\partial t}\left(a_{n+m}+a_{|n-m|}\right)=\left(\frac{\partial^{2} H_{n}}{\partial \xi^{2}}-n^{2} H_{n}\right)+Z H_{n}(\xi, t) \\
a_{o} \frac{\partial h_{n}}{\partial t}+\frac{1}{2} \sum_{1}^{N} \frac{\partial h_{m}}{\partial t}\left(a_{|m-n|}-a_{n+m}\right)=\left(\frac{\partial^{2} h_{n}}{\partial \xi^{2}}-n^{2} h_{n}\right)+Z h_{n}(\xi, t)
\end{gathered}
$$

where $a_{o}, a_{n}$ are the coefficients that result from expanding the term $\mathrm{J}$ in the form $\left(a_{o}+\sum_{n=1}^{\infty} a_{n} \cos n \eta\right)$. SGo, SGn, Sgn, $\mathrm{ZH}_{\mathrm{o}}, \mathrm{ZH}_{\mathrm{n}}$ and $\mathrm{Zh}_{\mathrm{n}}$ are all easily identifiable functions. Equations (10)-(12) define a system of differential equations that should be solved simultaneously at every time step.

The steady boundary conditions for all Fourier functions presented in equations (1012) are deduced from (8) and can be expressed as:

- At $\xi=\xi_{i}$

$\mathrm{f}_{\mathrm{n}}=\partial \mathrm{f}_{\mathrm{n}} / \partial \xi=\partial \mathrm{F}_{\mathrm{o}} / \partial \xi=\mathrm{F}_{\mathrm{n}}=\partial \mathrm{F}_{\mathrm{n}} / \partial \xi=0, \frac{\partial H_{o}}{\partial \xi}=2 B_{o}, \frac{\partial H_{n}}{\partial \xi}=B_{n}$ and $\frac{\partial h_{n}}{\partial \xi}=0$

- At $\xi=\xi_{0}$

$$
f_{n}=\partial f_{n} / \partial \xi=F_{0}=\partial F_{0} / \partial \xi=F_{n}=\partial F_{n} / \partial \xi=0, H_{n}=h_{n}=0 \text { and } H_{0}=0
$$

$B_{0}$ and $B_{n}$ are the coefficients in the expansion of the term $-J^{1 / 2}$ as

$\left(B_{o}+\sum_{n=1}^{\infty} B_{n} \cos n \eta\right)$

From the condition at inner wall (13b) it can be concluded that the value of stream function at the inner wall will be equal to $\psi_{\text {wall }}=F_{o \text { (wall })} / 2$. The value of $F_{\circ}$ at the wall is determined from the solution of eq. (10a). The vorticity distribution along the inner wall is found from the following integral conditions that developed from the integration of eq. (10) from $\xi=\xi_{\text {i }}$ to $\xi=\xi_{\text {o }}$ and using boundary conditions (13).

$$
\begin{gathered}
\int_{\xi_{i}}^{\xi_{o}}\left(a_{o} G_{o}+\sum_{1}^{N} a_{m} G_{m}\right) d \xi=0 \\
\int_{\xi_{i}}^{\xi_{o}}\left(\frac{1}{2} a_{n} G_{o}+a_{o} G_{n}+\frac{1}{2} \sum_{1}^{N} G_{m}\left(a_{n+m}+a_{|m-n|}\right)\right) e^{-n \xi} d \xi=0 \\
\int_{\xi_{i}}^{\xi_{o}}\left(a_{o} g_{n}+\frac{1}{2} \sum_{1}^{N} g_{m}\left(a_{|m-n|}-a_{n+m}\right)\right) e^{-n \xi} d \xi=0
\end{gathered}
$$


The above integrals are used to calculate the values of the functions $G_{0}, G_{n}, g_{n}$ on the inner wall surface from which the vorticity distribution along the wall is predicted. The vorticity distribution at outer wall is calculated at $\left(\xi_{=}=\xi_{0}\right)$ from eq. (10).

The numerical analysis proceeds further by discretizing the differential equation (1012) using finite difference method. In discretization process all spatial derivatives are approximated using second order accurate central finite difference scheme. The first derivatives at the inner and outer walls are approximated by one side second order accurate forward (at inner wall) or backward (at outer wall) three point finite difference scheme. The discretization of time dependent equations (11-12) is handled using Crank-Nicolson scheme. The forward time step is taken very small (less than 0.005) to achieve the accuracy of the scheme. At every time step the coupled non-linear system of equations has been linearized through an iterative procedure, resulting in tri-diagonal system of equations that has been solved by TriDiagonal Matrix Algorithm, TDMA. Badr and Dennis [23], and Mahfouz and Badr [24] give more details about solution procedure.

\section{Heat Transfer Parameters}

After obtaining the distributions of stream function and temperature, the flow and heat transfer characteristics can be easily determined. The stream function distribution facilitates plotting the flow field in terms of streamlines and also enables determining the flow velocity. While the temperature distribution helps find the average inner wall temperature and local heat flux on the outer wall. In case of CHF the total heat transfer rate from the inner wall is known and the most important parameter from practical point of view is the surface temperature of the inner wall, $\phi_{i}$ which should be inspected to avoid the unwanted over heating of the surface. The value of $\phi_{i}$ is calculated from (9c) and the average dimensionless temperature of the inner wall is then calculated as:

$$
\phi_{m i}=\frac{1}{p_{i}} \int_{0}^{P i} \phi_{i} d p_{i}
$$

For steady state pure conduction in case of CHF the analytical temperature distribution along the inner wall has been deduced as

$$
\phi_{i}\left(\xi_{i}, \eta\right)=X_{i o}+\sum_{1}^{\infty} \frac{B_{n}}{n} \tanh n X_{i o} \cos (n \eta)
$$

and the average temperature of the inner wall is then equal to

$$
\phi_{m i}=X_{i o}+\sum_{n=1}^{\infty} \frac{B_{n}^{2}}{2 n} \tanh n X_{i o}
$$

Similarly, the local heat flux distribution along the outer wall for steady pure conduction can be expressed as 


$$
q_{r}=q_{o} / q_{i}=-\frac{\cosh \left(\xi_{o}\right)-\cos (\eta)}{d}\left(-1+\sum_{1}^{\infty} \frac{B_{n} \cos (n \eta)}{\cosh n X_{i o}}\right)
$$

where $X_{i o}=\xi_{o}-\xi_{i}$.

The steady state solution is reached when the total heat ratio, $T H R=\dot{Q}_{o} / \dot{Q}_{i}$ $=\int q_{o} d p_{o} / q_{i} p_{i}$ approaches unity.

\section{RESULTS AND DISCUSSION}

Although the validity of mathematical model and numerical technique has been assessed in previous studies of Mahfouz [8, 17] and Mahfouz and Badr [23] , some more comparisons for the present case of $\mathrm{CHF}$ are considered. These comparisons are presented in Fig. 2. Figure 2a shows comparison with the work of Ho et al. [22] for distributions of both heat flux (in terms of $\mathrm{q}_{\mathrm{r}}$ ) along outer wall and the temperature along inner wall $\phi_{i}$ in case of natural convection in concentric annulus (e $\approx 0)$. While Fig. $2 \mathrm{~b}$ shows the same comparisons for $\mathrm{qr}$ and $\phi_{i}$ in case of symmetric eccentric annulus $(e=-0.625)$. The comparisons are in very good agreement with those of Ho.et al. [22] for both concentric and eccentric annuli. Once being satisfied with the validity and accuracy of the numerical solution, a set of runs is carried out to study the effect of controlling parameters on both flow and thermal fields within the annulus. The main controlling parameters beside the classical ones ( $R a L$ and $\operatorname{Pr}$ ) are the angle of inclination of ASL with respect to horizontal, $\lambda$ the eccentricity, e and the radius ratio, $\mathrm{Rr}$. The calculations were carried out at moderate $\mathrm{RaL}$ of $10^{4}$ and $\operatorname{Pr}=0.7$. The angle of $A S L \lambda$ is varied from $0^{\circ}$ to $90^{\circ}$ and the eccentricity is varied between -0.5 and +0.5 while three radius ratios $2,2.6,3.2$ are considered.

The numerical solution, as mentioned, has marched in time till reaching the steady state solution. The steady state solution is reached when the total heat rejected from the outer wall of the annulus approaches that pumped into the annulus through the inner wall. Fig.3 shows the time development of the total heat ratio $\left(T H R=\dot{Q}_{o} / \dot{Q}_{i}\right)$ along the outer wall when ASL is vertical $(\lambda=90)$ and when ASL is horizontal $(\lambda=0)$ at $\mathrm{RaL}=10^{4}, \mathrm{Rr}=2.6$ and $\mathrm{e}=+0.5$. The figure shows that the rate of heat rejected from outer wall $\dot{Q}_{o}$, increases with time till it becomes equal to that pumped through the inner wall $\dot{Q}_{i}$, at the steady state (i.e at THR=1). It can also be seen that in the very initial time stages the time development of THR is almost the same for the two cases of $\lambda=90$ and $\lambda=0$. This is because in initial time stages the heat transmission occurs only by conduction through very thin layer adjacent to inner wall and no role for convection.

The patterns of steady state streamlines and isotherms in case of symmetrical annulus $\lambda=90$ are shown in Fig. 4 while those in case of an asymmetric annulus $\lambda=$ 0 are shown in Fig. 5. It can be seen in symmetric case that both the flow field, Fig.4a, and the thermal field, Fig. $4 \mathrm{~b}$, are symmetric with respect to ASL. Fig. $4 \mathrm{a}$ shows that the flow field consists of two similar eddies one on each side of the 
annulus. These two eddies are separated by zero streamline line (dashed line) which coincides in this case with inner and outer walls and ASL line as well. In case of asymmetric field, Fig. $5 \mathrm{a}$, the two eddies are no longer being similar but rather have different sizes. In this case the zero streamline coincide with neither the inner wall nor the ASL but rather shifted away from the inner wall, indicating the existence of global flow circulation around the inner wall. The global flow was found to join the eddy in the larger side of the annulus. In this case the imbalance between induced flow in the two sides of the annulus creates a global flow that circulates around the inner wall as shown in the figure. Moreover, the thermal field in case of asymmetric case, Fig. 5b shows the formation and inclination of thermal plume in the direction of flow that comes upward and leaves the hot inner wall towards the cold outer wall. Moreover, the asymmetry of thermal field is further inspected in Fig. 6 which shows the heat flux distribution ( in terms of local $\mathrm{q}_{\mathrm{r}}$ ) along the outer wall of the annulus in case of $\lambda=90$ ( Fig. 6a) and in case of $\lambda=0$ (Fig. 6b). Unlike the symmetrical case ( $\lambda=90)$, Fig. $6 b$ shows that the distribution of heat flux in case of $\lambda=0$ is not symmetrical with respect to ASL.

Further inspection of Fig. 6a shows that the maximum heat flux $\left(\mathrm{qr}_{\mathrm{r}}\right)$ along the outer wall occurs in case of $\lambda=90$ at the point of flow stagnation at the wall at $\theta=0$ while the minimum $\mathrm{qr}$ occurs at $\theta \approx 100$. The figure also shows that the inner wall temperature $\phi_{i}$ assumes its highest value at the point of upward flow separation from the wall at $\theta=0$ and then decreases along the wall till it assumes its smallest value at almost $\theta \approx 115$. In case of $\lambda=0$ the inspection of Fig. $6 \mathrm{~b}$ shows that the maximum $\mathrm{qr}_{\mathrm{r}}$ occurs at the point of upward flow stagnation on the outer wall at $\theta \approx 84$ while the minimum value occurs at $\theta \approx 299$. The figure also shows that the inner wall temperature, $\phi_{i}$ is maximum at the point of upward flow separation from the inner wall at $\theta \approx 81$ while the smallest value occurs at . $\theta \approx 335$

Table 1 shows the effect of controlling parameters on $\phi_{m i}$ at $\mathrm{Rr}=2.4$. Shown also in the table are the numerical and analytical values for steady average inner wall temperature in case of pure conduction $(R a L=0)$. It can be seen that in case of $\mathrm{RaL}=0$ both numerical and analytical values are almost the same which further assesses the accuracy of the numerical solution. In case of considering convection effects $\left(\mathrm{RaL}=10^{4}\right)$, the table shows that $\phi_{m i}$ decreases for all inclinations and eccentricities in comparison with corresponding pure conduction values as a result of increasing convection heat transfer coefficient.

The effect of eccentricity e on $\phi_{m i}$ can be also observed in Table 1 . In case of RaL=0 the table shows that $\phi_{m i}$ is maximum in case of $\mathrm{e} \approx 0$ (i.e almost concentric annulus) and decreases as the absolute value of e increases (from 0 to 1). In case of considering convection effects the table shows that the $\phi_{m i}$ in case of any negative eccentricity is lower than that for same but positive eccentricity. This is because the negative eccentricity configuration is more favorable for heat convection process which increases the heat transfer coefficient at the inner wall and thus reduces the average wall temperature.

Table 1 further shows the effect of ASL inclination angle $\lambda$ on average inner wall temperature $\phi_{m i}$. It can be seen that as $\lambda$ increases $\phi_{m i}$ decreases in case of 
negative eccentricities while it increases in case of positive eccentricities. In case of negative eccentricity as the angle of ASL increases from 0 to 90 the upper gap between the two walls gets wider and so enhances heat convection process and thus increases the average heat transfer coefficient at the inner wall. While for same but positive eccentricity as the angle of ASL increases the upper gap between the two walls gets narrower which reduces the average heat transfer coefficient at the inner wall and thus increases $\phi_{m i}$.

Table 2 shows the effect of $\operatorname{Rr}$ on $\phi_{m i}$ at $\lambda=60$. The table shows that as $\operatorname{Rr}$ increases the $\phi_{m i}$ increases as a result of poor convection process. The table also shows that as eccentricity e moves from negative to positive values, $\phi_{m i}$ increases. It seems for this moderate value of Ral the role of convection is not significant and conduction is almost controlling heat transfer process in the annulus. As $\mathrm{Rr}$ increases the conduction resistance increases which reduces the heat transfer coefficient and thus increasing the inner wall average temperature $\phi_{m i}$.

The flow field and thermal fields are shown in Fig. 7 for the cases of asymmetric annulus at $\lambda=0,30$, and 60 . The figure shows the existence of global flow circulation around the inner wall. The intensity of global flow can be inferred from the position of zero stream function line with respect to the inner wall. The closer the line to the inner wall the less the intensity of global flow. Moreover, the figure shows that the global flow joins the left hand eddy in this case of positive eccentricities, which is unlike the case of negative eccentricity where the global flow joins the right hand eddy ( see Fig. 5a). The effect of increasing global flow intensity on the inclination of thermal plume above the inner wall can be also observed. The more the intensity of global flow the more the inclination of thermal plume in the direction of global flow circulation.

\section{CONCLUSIONS}

In this work, the natural heat convection within an annulus formed between two eccentric long cylinders has been studied. The annulus inner wall is heated and maintained at constant heat flux while the outer wall is cooled and maintained at constant temperature. Beside Rayleigh and Prandtl numbers the heat convection process in the annulus depends on the inclination of ASL, radius ratio and eccentricity. The study considered a moderate Rayleigh number of $10^{4}$ while Prandtl number is fixed at 0.7 . The angle of ASL is varied from zero (the horizontal position) to 90 (vertical position). The study is carried out at three values of radius ratio namely, 2, 2.6, 3.2, while the eccentricity is varied between \pm 0.5 . The study has shown that as $\lambda$ increases from 0 to 90 the average inner wall temperature $\phi_{m i}$ decreases for negative eccentricities while it deceases for positive eccentricities. The study also shows that as $\mathrm{Rr}$ increases from 2 to 3.2 the $\phi_{m i}$ increases. The study has also shown that the present numerical solution of the pure conduction problem is almost identical with the analytical solution which confirms the high accuracy of the numerical solution. 


\section{ACKNOWLEDGMENT}

The support received from Menoufia University to carry out this piece of work is highly appreciated.

\section{REFERENCES}

[1] Kuehn T. H. and Goldstein, R. J., "An Experimental and Theoretical Study of Natural Convection in the Annulus between Horizontal Concentric Cylinders," J. Fluid Mech., 74, pp. 695-719, (1976).

[2] Kuehn T. H. and Goldstein, R. J., "An Experimental Study of Natural Convection Heat Transfer in Concentric and Eccentric Horizontal Cylindrical Annuli," ASME J. Heat Transfer, 100, pp. 635-640, (1978).

[3] Chakrabarti, S., Probert, S. D. and Shilton, M. J., "Optimal Eccentric Annuli (containing atmospheric-pressure air) for Thermally Insulating, Horizontal, Relatively Cold Pipes", Appl. Energy, vol. 14, pp. 257-293, (1983).

[4] Prusa, J., and Yao, L. S., "Natural Convection Heat Transfer Between Eccentric Horizontal Cylinders,” ASME, J. Heat Transfer 105, pp. 108-116, (1983).

[5] Habibi, M. M., and Pop, I., "Natural convection flow and heat transfer in an eccentric annulus filled by Copper nanofluid", Int. J. Heat and Mass Transfer,61 , pp. 353-364, (2013)

[6] Guj, G. and Stella, F., "Natural Convection in Horizontal Eccentric Annuli: Numerical Study," Num. Heat Transfer 27, pp. 89-105, (1995).

[7] Guj G., lannetta S. and Moretti G., "Experimental Analysis of Thermal Fields in Horizontal Eccentric Cylindrical Annuli", Exp. Fluid, vol. 12, pp. 385-393, (1992).

[8] Mahfouz, F. M., "Buoyancy Driven Flow within an Inclined Elliptic Enclosure", Int. J. of Thermal Sciences, 50, pp. 1887-1899, (2011).

[9] Wang, Y. Z. and Bau, H. H., "Low Rayleigh Number Convection in Horizontal, Eccentric Annuli", Phys. Fluids, 31(9), pp. 2467-2473, (1988).

[10] Cho, C. H., Chang, K.S., and Park, K.H., "Numerical Simulation of Natural Convection in Concentric and Eccentric Horizontal Annuli," ASME, J. Heat Transfer 104, pp. 624-630, (1982).

[11] Projahn, U., and Beer, H., "Prandtl Number Effects on Natural Convection Heat Transfer in Concentric and Eccentric in Horizontal Cylindrical Annuli," Heat and Mass Transfer, 19, 249-254, (1985).

[12] Adlam, J. H., "Computation of two-Dimensional Time-Dependent Natural Convection in a Cavity Where There Internal Bodies", Compt. Fluids, 14, pp. 141-157, (1986).

[13] Stella, F. and Guj, G., "Vorticity-Velocity Formulation in the Computation of Fluids in Multiconnected Domains", Int. J. Num. Methods Fluids, 9, pp. 12851298, (1989).

[14] Shu, C., Yao, Q., Yeo, K.S, "An Efficient Approach to Simulate Natural Convection in Arbitrarily Eccentric Annuli by Vorticity-Stream Function Formulation". Numer. Heat Transfer, A 38, 739-756, (2000). 
[15] Shu, C., and Wu, Y.L., "Domain-Free Discretization Method for Doubly Connected Domain and its Application to Simulate Natural Nonvection in Eccentric Annuli," Comput. Methods Appl. Mech. Eng., 191, pp. 1827-1841, (2002)

[16] Lee, T. S., Hu, G. S., and Shu, C., "Application of GDQ Method for the Study of Natural Convection in Horizontal Eccentric Anuuli," Num. Heat Transfer, Part A, 4, pp. 803-815. (2002).

[17] Mahfouz, F. M, Natural Convection Within an Eccentric Annulus at Different Orientations, AIAA, Journal of Thermophysics and Heat Transfer , 26(4), pp. 665-672, (2012)

[18] Castrejon, A., and Spalding, D.B., "An Experimental and Theoretical Study of Transient Free-Convection Flow between Horizontal Concentric Cylinders," Int. J. Heat Mass Transf. 31 (2), pp. 273-284. (1988)

[19] Yoo, J.- S., "Flow Pattern Transition of Natural Convection in a Horizontal Annulus with Constant Heat Flux on the Inner Wall," Int. J. of Num. Methods for Heat and Fluid Flow, 15(7), pp. 698-709, ( 2005)

[20] Yoo, J.- S., "Dual Free-Convective Flows in a Horizontal Annulus with a Constant Heat Flux Wall". Int J. Heat and Mass Transfer, 46, pp. 2499-2503, ( 2003)

[21] Glakpe, E.K., Watkins Jr., C.B., and Cannon, J. N., "Constant Heat Flux Solutions for Natural Convection between Concentric and Eccentric Horizontal Cylinders," Num. Heat Transf. 10 (3), pp. 279-295, (1986)

[22] Ho, C. J., Lin, Y. H., and Chen, T. C., "A Numerical Study of Natural Convection in Concentric and Eccentric Horizontal Cylindrical Annuli with Mixed Boundary Conditions," Int. J. Heat and Fluid Flow, 10(1), pp. 40-47. (1989)

[23] Badr, H. M., and Dennis, S. C. R., "Time-Dependent Viscous Flow Past an Impulsively Started Rotating and Translating Circular Cylinder," J. Fluid Mech. 158, pp. 447-488, (1985).

[24] Mahfouz, F. M. and Badr, H. M., "Flow Structure in the Wake of a Rotationally Oscillating Cylinder," ASME J. of Fluids Eng., 122, pp. 290-301, (2000). 


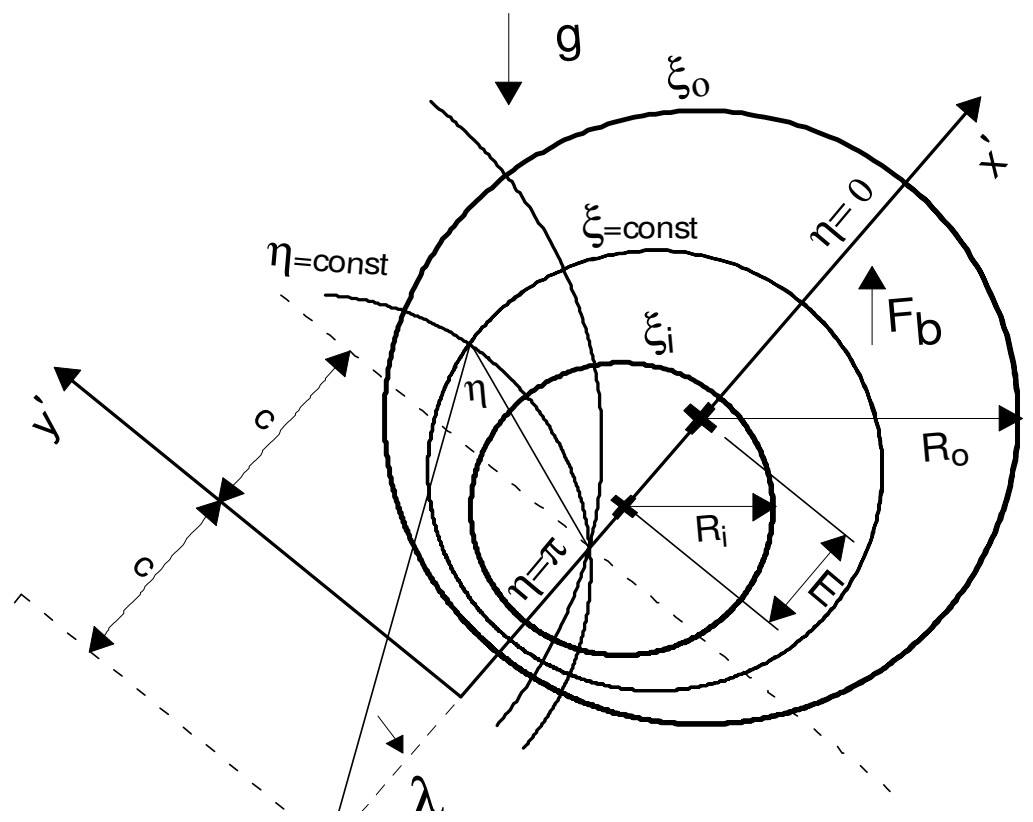

Fig. 1. Physical domain and coordinate systems

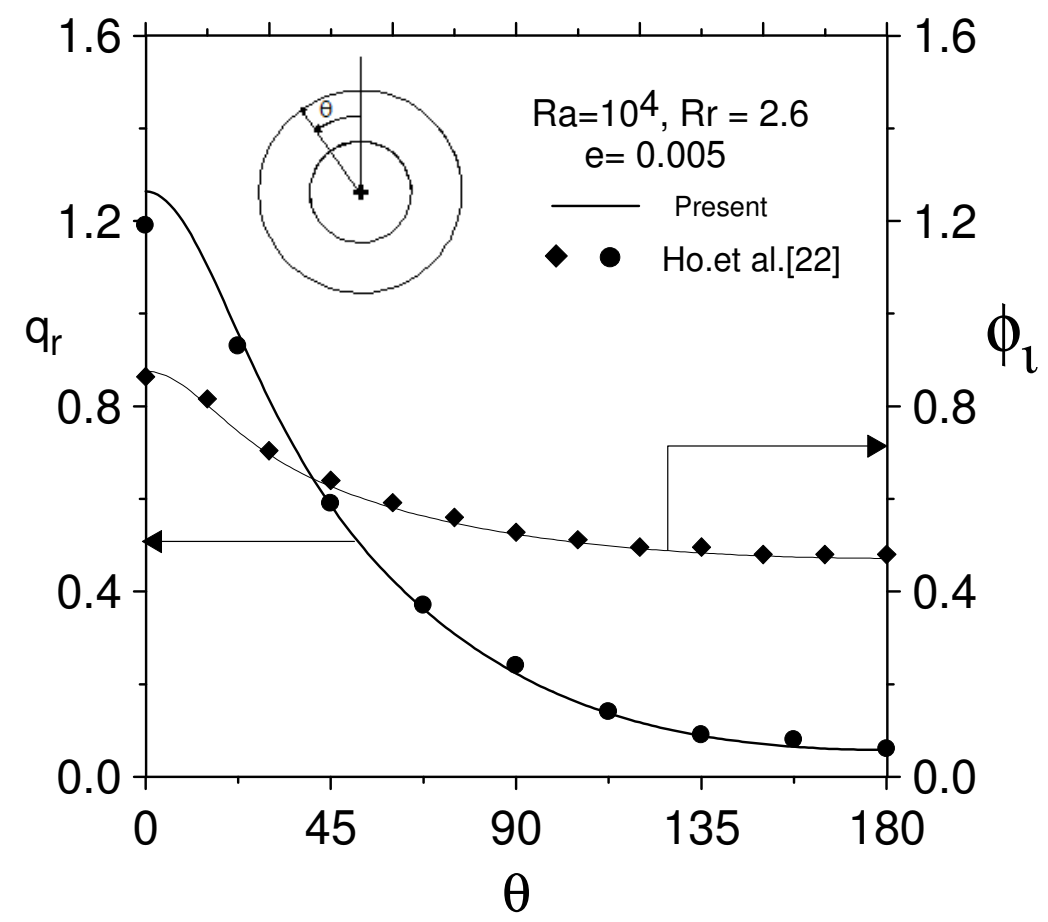

Fig.2a. Distribution of $\mathrm{q}_{\mathrm{r}}$ along the outer wall and $\phi_{i}$ along the inner wall and comparison with $\mathrm{Ho}$ et al. [22] at $\mathrm{RaL}=10^{4}, \mathrm{Rr}=2.6$, and $\mathrm{e} \approx 0.0$. 


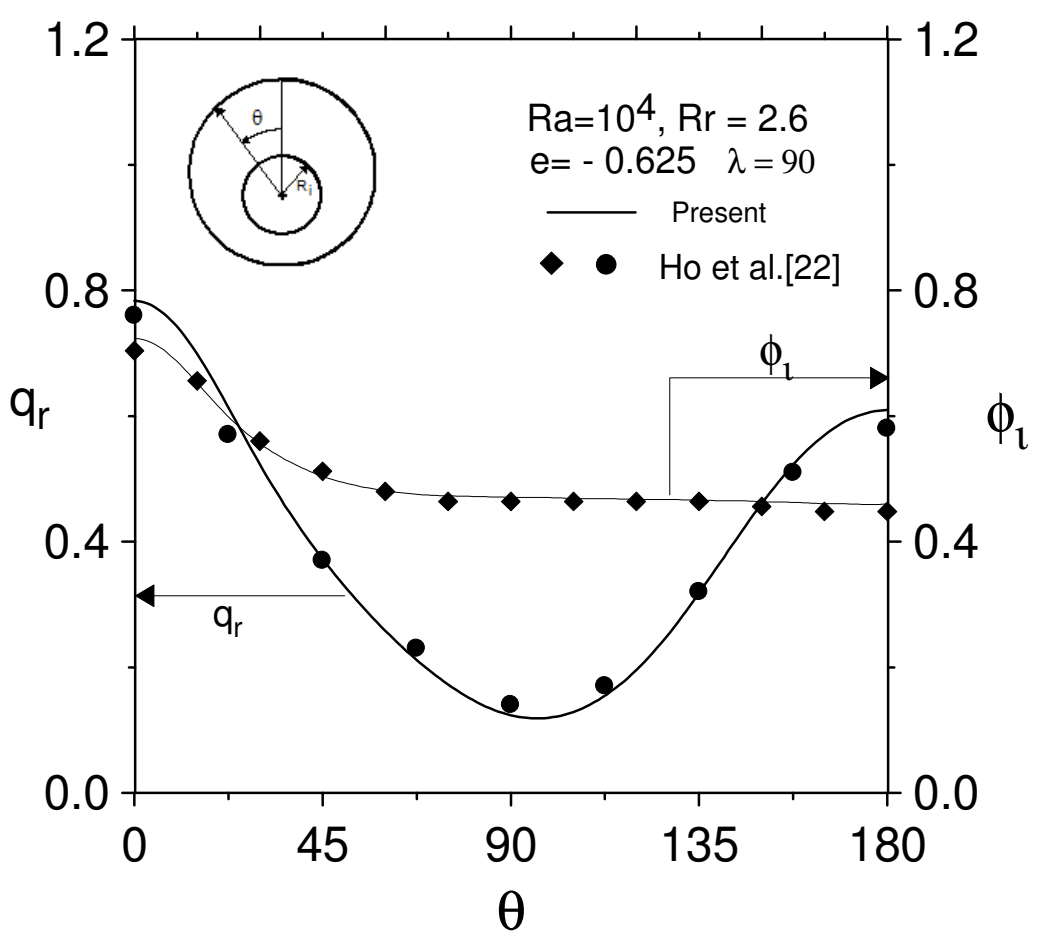

Fig.2b. Distribution of $\mathrm{qr}_{\mathrm{r}}$ along the outer wall and $\phi_{i}$ along the inner wall and comparison with $\mathrm{Ho}$ et al. [22] at $\mathrm{RaL}=10^{4}, \mathrm{Rr}=2.6$, and $\mathrm{e}=-0.625$

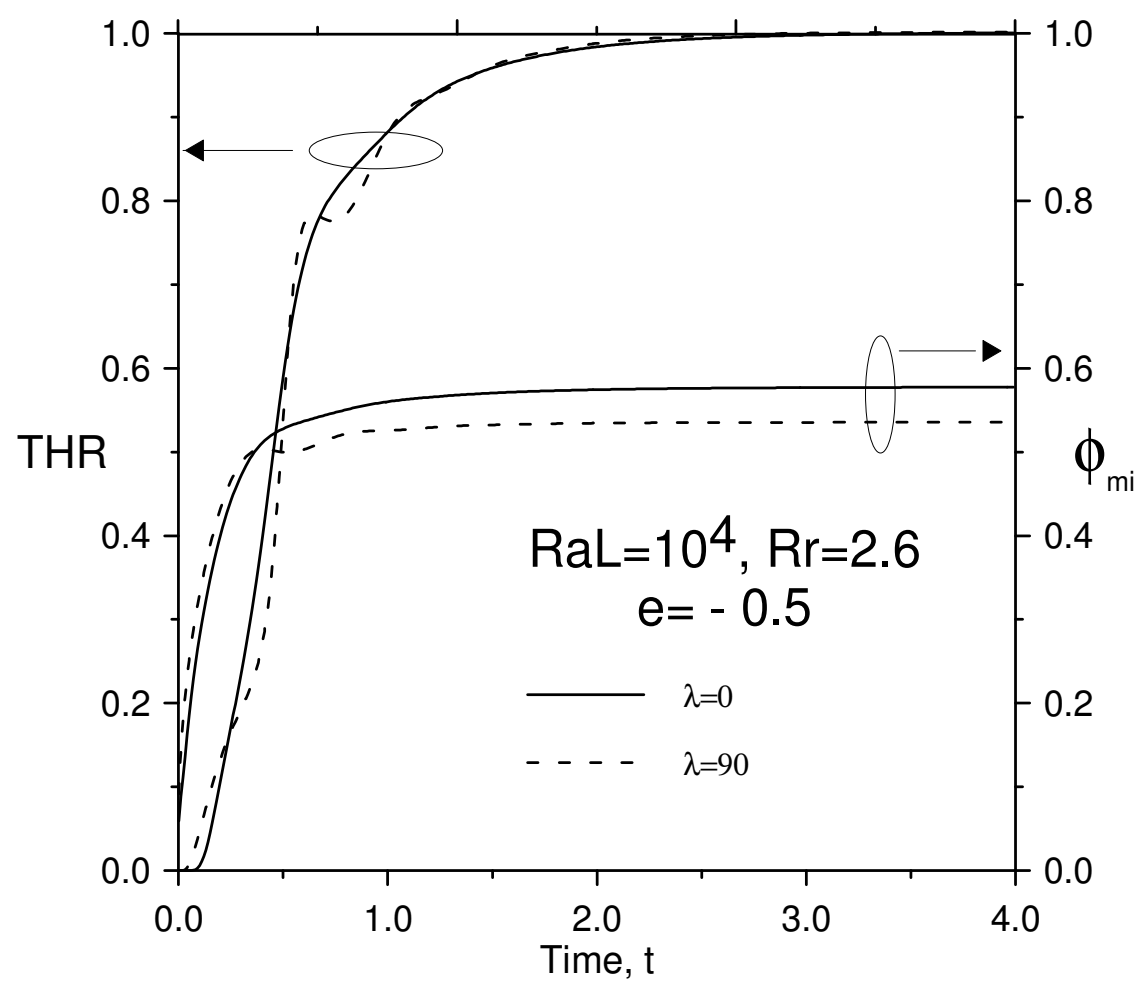

Fig. 3. The time development of $\mathrm{THR}$ and $\phi_{m}$ at $\mathrm{RaL}=10^{4}, \mathrm{e}=-0.5, \mathrm{Rr}=2.6$ and at ASL vertical $(\lambda=90)$ and ASL horizontal $(\lambda=0)$ 


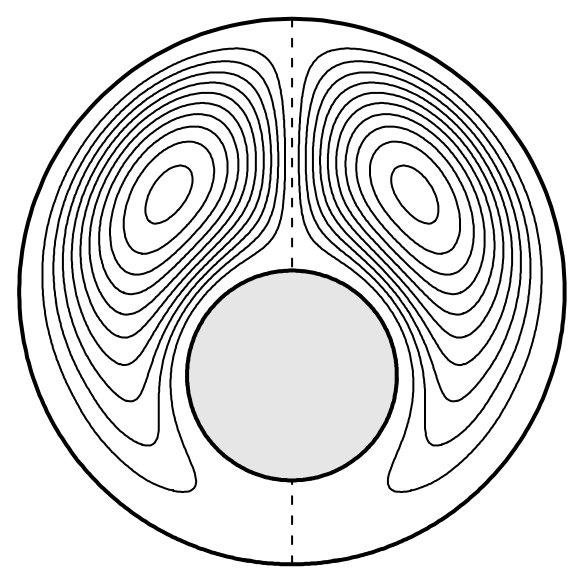

(a)

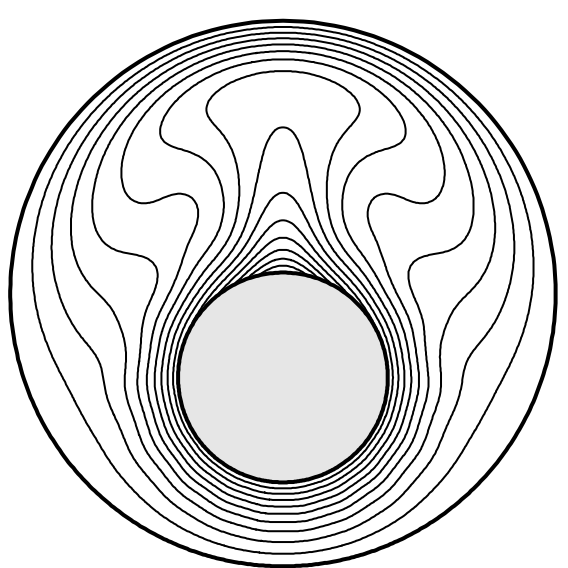

(b)

Fig.4. The Streamlines (left) and isotherms (right) for case of Ral=104, $\mathrm{e}=-0.5$ and $\lambda=90.0$

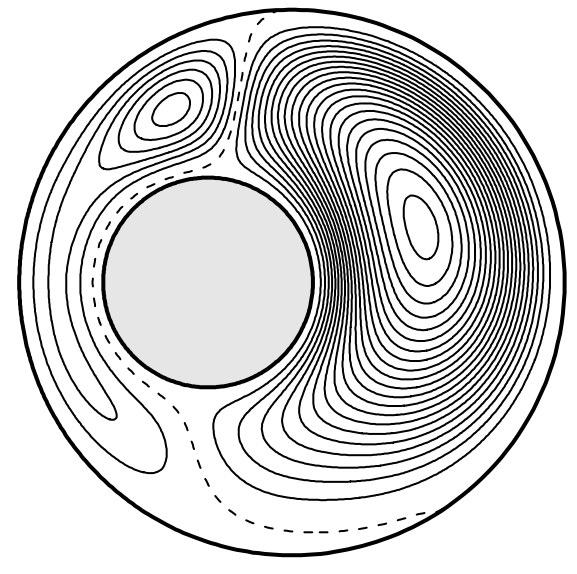

(a)

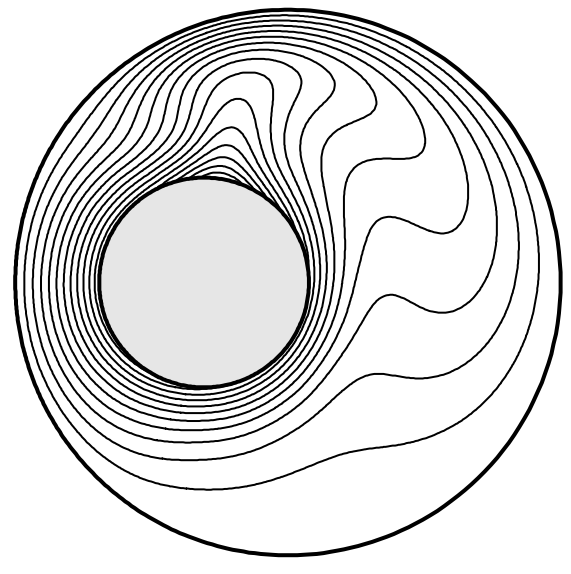

(b)

Fig.5. The Streamlines (left) and isotherms (right) for case of $\mathrm{Ral}=10^{4}, \mathrm{e}=-0.5$ and $\lambda=0.0$ 


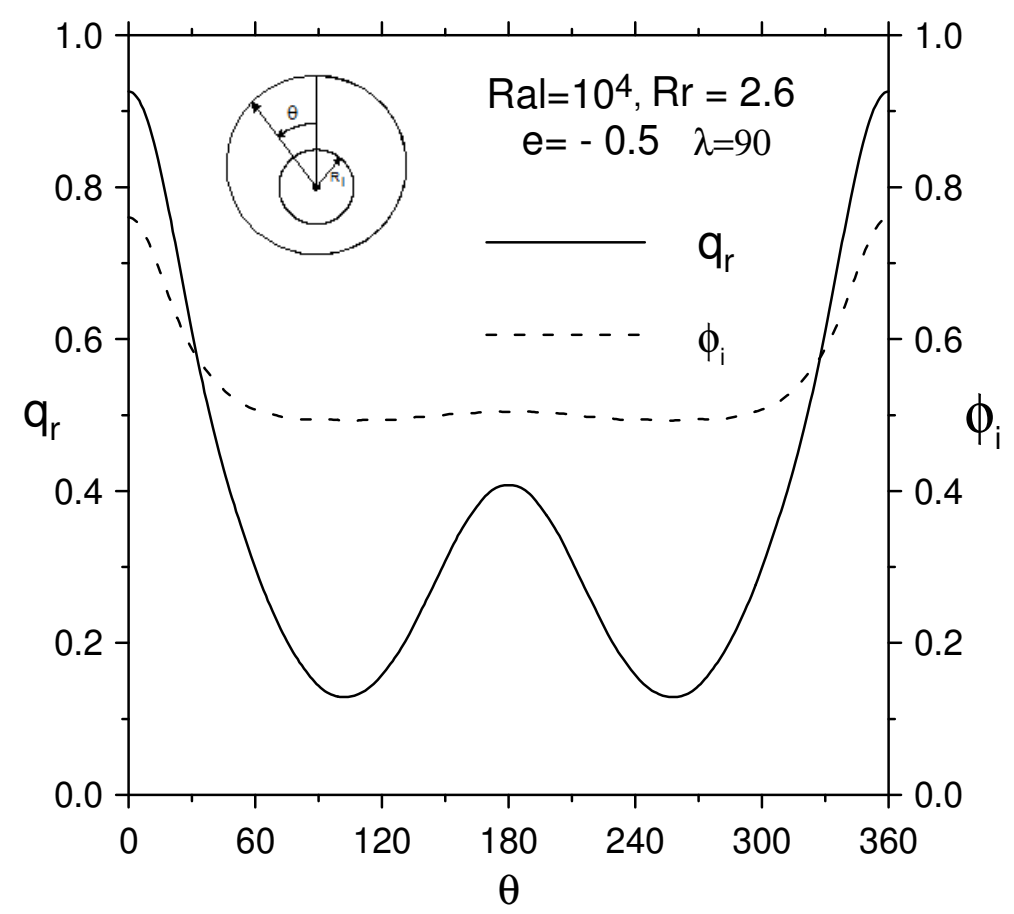

(a)

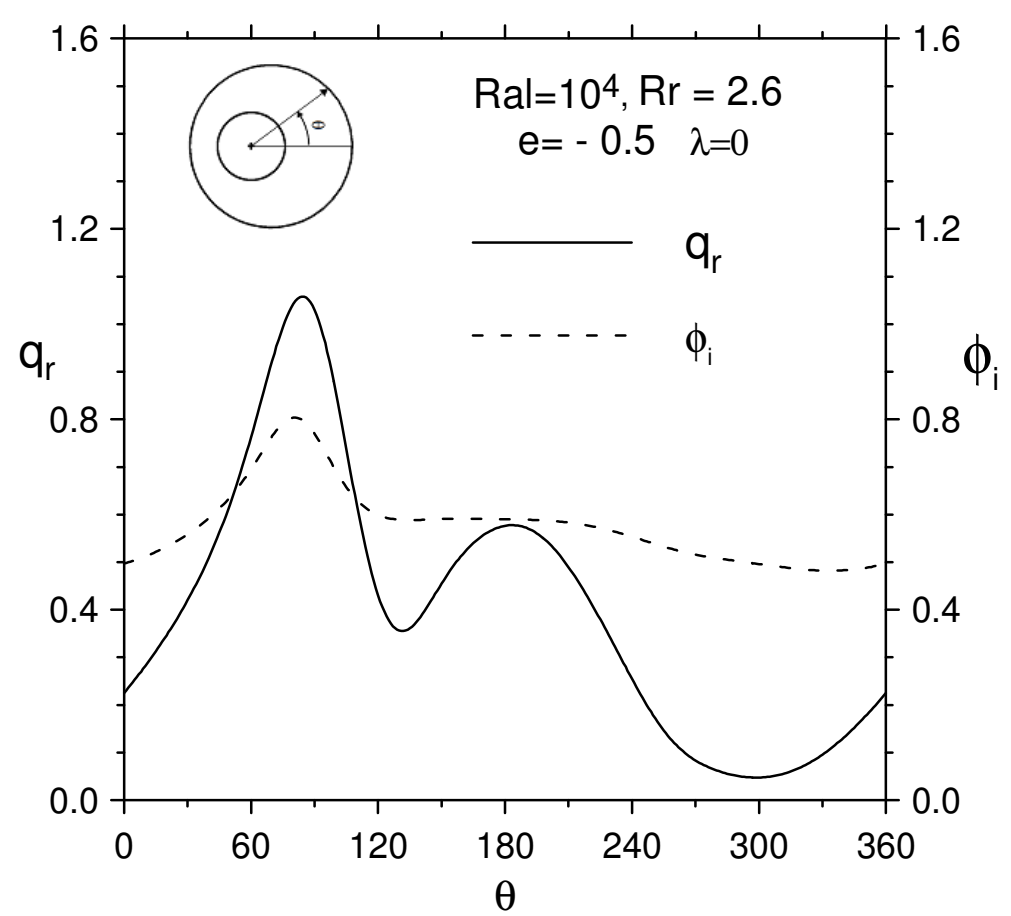

(b)

Fig. 6. Local distributions of inner wall temperature and outer wall heat flux at $\mathrm{RaL}=$ $10^{4}, \mathrm{e}=-0.5$ and a) $\lambda=90$ and b) $\lambda=0$ 
a) $\lambda=0$
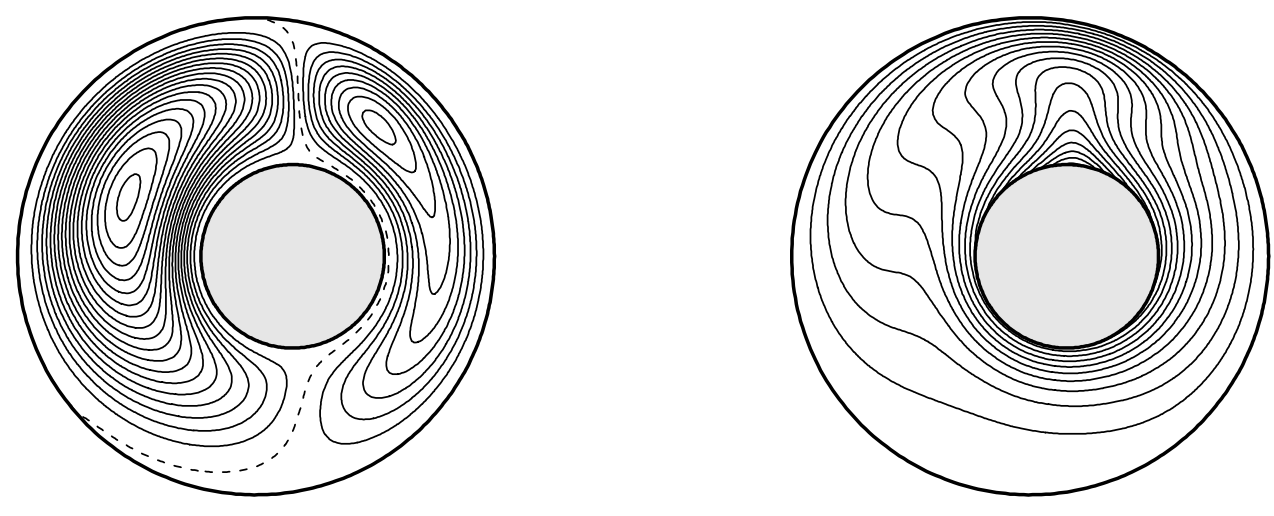

b) $\lambda=30$
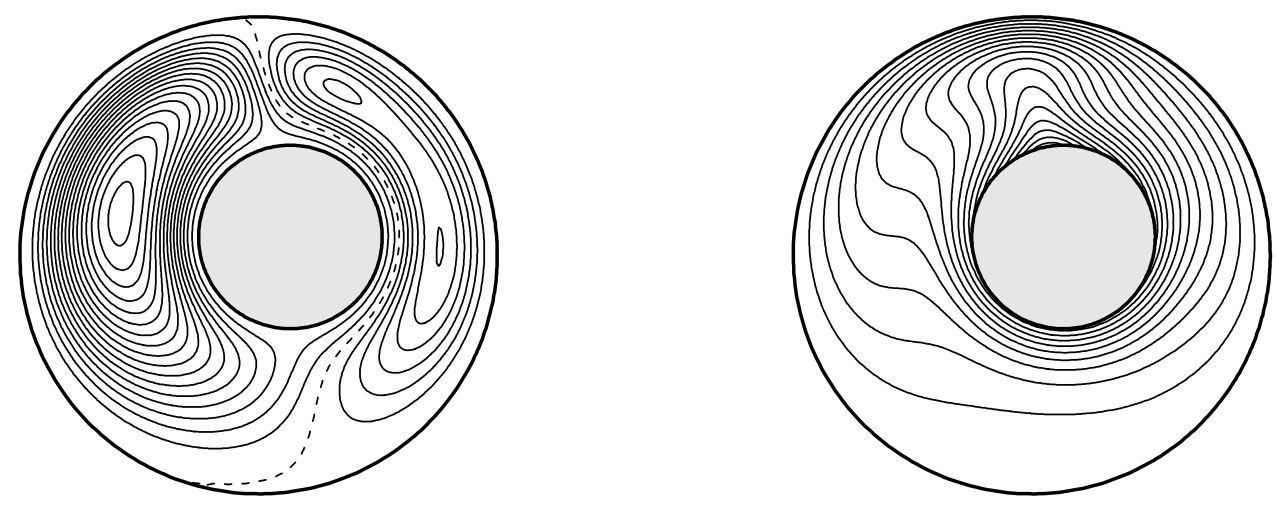

c) $\lambda=60$
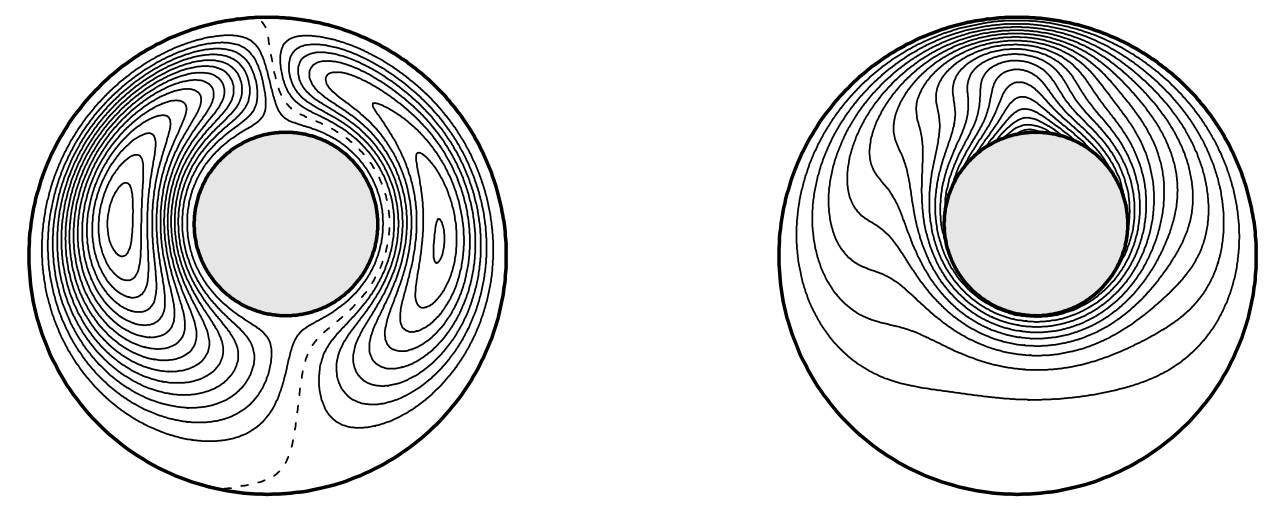

Fig. 7. The Streamlines (left) and isotherms (right) at $\mathrm{RaL}=10^{4}, \mathrm{e}=+0.25$ and at a) $\lambda=0 \quad$ b) $\lambda=30$ and c) $\lambda=60$ 
Table 1. Effect of $\lambda$ and e on steady $\phi_{m i}$ at $\mathrm{Rr}=2.4$.

\begin{tabular}{|c|c|c|c|c|c|c|}
\hline \multirow{3}{*}{ RaL } & \multirow{2}{*}{$\lambda$} & \multicolumn{5}{|c|}{$\phi_{m i}$} \\
\cline { 3 - 7 } & & $\mathrm{e}=-0.50$ & $\mathrm{e}=-0.25$ & $\mathrm{e} \approx 0.0$ & $\mathrm{e}=+0.25$ & $\mathrm{e}=+0.50$ \\
\hline \hline \multirow{2}{*}{0} & & 0.8011 & 0.8571 & 0.8752 & 0.8571 & 0.8011 \\
& & $(0.8012)^{*}$ & $(0.8572)^{*}$ & $(0.8754)^{*}$ & $(0.8572)^{*}$ & $(0.8012)^{*}$ \\
\hline \hline \multirow{4}{*}{$10^{4}$} & 0 & 0.5272 & 0.5325 & 0.5258 & 0.5325 & 0.5272 \\
\cline { 2 - 7 } & 30 & 0.5069 & 0.5248 & 0.5258 & 0.5400 & 0.5418 \\
\cline { 2 - 7 } & 60 & 0.4938 & 0.517 & 0.5258 & 0.5477 & 0.5585 \\
\cline { 2 - 7 } & 90 & 0.4893 & 0.5142 & 0.5258 & 0.5505 & 0.5631 \\
\hline
\end{tabular}

${ }^{*}$ Analytical $\phi_{m i}$

Table 2. Effect of $\mathrm{Rr}$, and e on steady $\phi_{m i}$ at $\lambda=60$.

\begin{tabular}{|c|c|c|c|c|c|c|}
\hline \multirow{3}{*}{$\operatorname{RaL}$} & \multirow{2}{*}{$\operatorname{Rr}$} & \multicolumn{5}{|c|}{$\phi_{m i}$} \\
\cline { 3 - 7 } & & $\mathrm{e}=-0.50$ & $\mathrm{e}=-0.25$ & $\mathrm{e} \approx 0.0$ & $\mathrm{e}=+0.25$ & $\mathrm{e}=+0.50$ \\
\hline \multirow{3}{*}{$10^{4}$} & 2.0 & 0.392 & 0.410 & 0.421 & 0.438 & 0.436 \\
\cline { 2 - 7 } & 2.6 & 0.539 & 0.563 & 0.573 & 0.596 & 0.608 \\
\cline { 2 - 7 } & 3.2 & 0.671 & 0.692 & 0.702 & 0.736 & 0.753 \\
\hline
\end{tabular}

